\title{
RESEARCH PAPER \\ Selection of rice genotypes (Oryza sativa) with high nitrogen agronomic efficiency in an Acuic Durixererts soil, central-southern Chile
}

\author{
Celerino Quezada, Santiago Hernaíz, Iván Vidal, Roberto Alvarado, Rubén \\ Gallegos, and Wilson Yañez \\ Departamento de Suelos y Recursos Naturales, Facultad de Agronomía, Universidad de Concepción. \\ Casilla 537. Chillán, Chile.
}

\begin{abstract}
C. Quezada, S. Hernaíz, I. Vidal, R. Alvarado, R. Gallegos, and W. Yañez. 2013. Selection of rice genotypes (Oryza sativa) with high nitrogen agronomic efficiency in an Acuic Durixererts soil, central-southern Chile. 40(2): 375-385. Nitrogen agronomic efficiency (NAE) is a key factor that has not been considered in rice breeding programs. The objective of this study was to evaluate nitrogen agronomic efficiency in twenty rice genotypes in an Acuic Durixererts soil (Quella Series) located in Chile's south central zone $\left(36^{\circ} 04^{`} \mathrm{~S}, 72^{\circ} 0^{\circ} \mathrm{W}\right.$ ) over the 2008-09 growing season. The field experiment was carried out in a complete randomized split-plot block design with five $\mathrm{N}$ treatments of $0,50,100,150$ and $200 \mathrm{~kg} \mathrm{ha}^{-1}$ and twenty genotype sub-treatments. The following agronomic characteristics of the crops were measured: yield, grain fill percentage, and nitrogen agronomic efficiency. The $\mathrm{N}$ level had a significant effect on grain yield, NAE and the number of panicles per $\mathrm{m}^{2}$. Highly significant differences in all parameters evaluated were observed between genotypes. The nitrogen $\mathrm{x}$ genotype interaction had significant effects on plant height, 1000-grain weight and the grain fill percentage. The best performing rice genotypes were as follows: Quila 233006, Quila 223202, CINIA 857, and RQuila 17 with a $50 \mathrm{~kg} \mathrm{~N}^{-1}$ dose and Ámbar, RQuila 17 and Quila 242105 with $100 \mathrm{~kg} \mathrm{~N}^{-1}$ dose. These genotypes had high NAE and yield components that may be of use when producing new cultivars. Our results demonstrate the importance of selecting rice genotypes with greater $\mathrm{NAE}$ and considering this parameter when making $\mathrm{N}$ fertilizer recommendations.
\end{abstract}

Key words: Nitrogen agronomic efficiency, nitrogen dose, rice genotypes.

\section{Introduction}

Nitrogen (N) is an essential nutrient for rice production. $\mathrm{N}$ is required during the vegetative

Received August 18, 2012. Accepted March 15, 2013. Corresponding author: cequezad@udec.cl stage to promote growth and tillering, which determines the number of panicles (Samonte et al., 2006). $\mathrm{N}$ is also as substrate for starch and protein synthesis during grain development (Jiang et al., 2004). Excessive application of $\mathrm{N}$ can have negative effects on sustainable agricultural development (Jian-feng et al., 2011). Rice breed- 
ers do not consider nitrogen agronomic efficiency (NAE) as a selection criterion when growing new crops, even though this trait has both economic and ecological implications (Samonte et al., 2006).

Nitrogen use efficiency in cereal production worldwide is approximately $33 \%$; this is due to the loss of $\mathrm{N}$ fertilizer from gaseous plant emissions, soil denitrification, surface runoff, volatilization and leaching (Raun and Johnson, 1999; Zhao et al., 2012). The high input rate and the improper timing of $\mathrm{N}$ application by farmers results in a low NAE for irrigated rice in China (Peng et al., 2006). Continued efforts are required to select for plants under low N, which is not often considered a priority by plant breeders (Raun and Johnson, 1999).

The yield increase that results from $\mathrm{N}$ application is defined as the NAE ( $\mathrm{kg}$ increase in grain per $\mathrm{kg} \mathrm{N}$ applied). The plant response to fertilizer is the result of genetic characteristics and crop management (Peng et al., 2006). Timsina et al. (2001) emphasized that to develop cultivars for productivity and $\mathrm{N}$-use efficiency, it is necessary to monitor weather and measure crop performance, water irrigation, and soil and plant mineral N. The translocation, distribution and remobilization of absorbed $\mathrm{N}$ in different plant organs must also be understood (Ladha et al., 1998; Jiang et al., 2004). Increasing NAE depends on the fieldspecific management of $\mathrm{N}$ and the use of systems that are responsive to soil $\mathrm{N}$ supply and plant $\mathrm{N}$ status (Cassman et al., 1998).

NAE is determined by the indigenous nutrient supply, the application of fertilizer, crop management practices and climate conditions. According to Tirol-Padre et al. (1996), efficient genotypes are those that produce high grain yields at suboptimal $\mathrm{N}$ levels through increased $\mathrm{N}$ uptake and/or more efficient $\mathrm{N}$ utilization. For cereals in developing countries, NAE ranged between 10 and $30 \mathrm{~kg} \mathrm{~kg}^{-1}$ and could reach $>25 \mathrm{~kg} \mathrm{~kg}^{-1}$ in a well-managed system with low levels of $\mathrm{N}$ use (Chuan et al., 2013). Cassman et al. (1998) found that Asian farmers in irrigated rice systems achieved an NAE of $15.3 \mathrm{~kg} \mathrm{~kg}^{-1}$ with standard practices and reached $23.7 \mathrm{~kg} \mathrm{~kg}^{-1}$ with improved nitrogen management using a chlorophyll meter.

The global demand for $\mathrm{N}$ fertilizer is consistently increasing, driven mainly by cereal production. Globally, approximately $40 \%$ of the human population relies on $\mathrm{N}$ fertilizer for food production. The use of such a large amount of $\mathrm{N}$ fertilizer takes a heavy toll on the health of the environment because the average NAE in an agricultural field is 33\% (Ahmad et al., 2008). An increase in grain yield and grain nitrogen use efficiency is an important objective of many rice breeding programs (Wei et al., 2011; Haefele et al., 2008). It is also important to obtain the greatest grain yield potential in rice crops (Jiang et al., 2004) and reduce $\mathrm{N}$-fertilizer requirements to protect the environment (Ladha et al., 1998).

Chilean rice crops are cultivated in a permanent $10-20 \mathrm{~cm}$ depth flood system. This system results in significant $\mathrm{N}$ losses by leaching, denitrification and volatilization; volatilization alone accounts for a 30-40\% loss of applied N (Fillery et al., 1986). To ensure an adequate supply of $\mathrm{N}$ throughout the growing season, $\mathrm{N}$ is applied as urea into floodwater during sowing, tillering and panicle initiation. Khurana et al. (2008) state that site-specific nutrient management leads to an increase in NAE.

Low rice fertility in soils and the high cost of fertilizer make it necessary to select rice crops that not only produce high yield but also use $\mathrm{N}$ efficiently, thereby producing grains with higher protein concentration (Samonte et al., 2006). An increase in cereal NAE can be obtained by using varieties with a high harvest index (Raun and Johnson, 1999), applying partial N, and further selecting stable genotypes under different nitrogen environments (Duan et al., 2007). The objective of this study was to evaluate performance, yield components and agronomic characteristics in an Aquic Durixererts soil to identify crops with high NAE. 


\section{Materials and methods}

\section{Site description}

A field experiment was carried out at the Digua Experimental Station $\left(36^{\circ} 04^{`} \mathrm{~S}, 72^{\circ} 0{ }^{\prime} \mathrm{W}, 140\right.$ masl) in Parral, Chile during the 2008-09 growing season. This soil is classified as very fine, smectitic, thermic Aquic Durixererts (Quella Series) and is derived from volcanic tuff. The soil is shallow (50 cm-depth) with poor drainage and low run off. The soil texture is classified as clay with an angular blocky structure and dark gray color, and the topography is nearly level and undulating (Stolpe, 2006). The soil had a $\mathrm{pH}$ of 5.76 and contained the following components: organic matter (2.34\%), nitrogen $\left(7 \mathrm{mg} \mathrm{kg}^{-1}\right)$, phosphorous $\left(6.61 \mathrm{mg} \mathrm{kg}^{-1}\right)$, potassium $(62.7 \mathrm{mg}$ $\left.\mathrm{kg}^{-1}\right)$ and incubated nitrogen $\left(10 \mathrm{mg} \mathrm{kg}^{-1}\right)$. The climate is Warm Mediterranean with a $983 \mathrm{~mm}$ annual rainfall; approximately 65 to $70 \%$ of the precipitation occurs between May and August. The annual potential evapotranspiration is 840 $\mathrm{mm}$, with a 4- to 5-month dry period and 4 to 5 frost-free months. The annual temperature is 14.2 ${ }^{\circ} \mathrm{C}$; the coldest month (July) has a temperature of $4.2{ }^{\circ} \mathrm{C}$, and the hottest month (January) has a temperature of $29.5{ }^{\circ} \mathrm{C}$. The average annual relative humidity is $62.7 \%$ (Del Pozo and Del Canto, 1999).

\section{Experimental design}

The field experiment was carried out using a completely randomized split-plot block design, consisting of five treatments ( $\mathrm{N}$ doses), twenty sub-treatments (rice genotypes) and four replications. The following $\mathrm{N}$ doses were used: 0 , $50,100,150$ and $200 \mathrm{~kg} \mathrm{ha}^{-1}$. The following rice genotypes were studied: Diamante, Brillante, Ámbar, Zafiro, Cuarzo, RQuila 28, CINIA 857, Dmu 81m, Quila 201704, Quila 223202, Quila 242415, Quila 233006, Quila 233008, Quila 242101, Quila 242105, RQuila 17, Quila 199604, Quila 157302, Quila 121304 and Quila 156906. Plot sizes were
$57.4 \mathrm{~m}^{2}(8.2 \times 7.0 \mathrm{~m})$ in the main plots and 1.21 $\mathrm{m}^{2}(1.1 \times 1.1 \mathrm{~m})$ in the sub-plots. The total area of the field experiment was $1,091 \mathrm{~m}^{2}$.

\section{Crop management}

Rice was sowed on October $29^{\text {th }}$ on a soil flooded with 5 -cm water using $160 \mathrm{~kg} \mathrm{ha}^{-1}$ of pre-germinated seed per dose. $\mathrm{N}$ fertilizer in the form of urea was applied as follows: $1 / 3$ was applied during sowing, $1 / 3$ was applied during tillering and $1 / 3$ was applied at panicle initiation. At sowing, phosphorous (100 $\mathrm{kg} \mathrm{P}_{2} \mathrm{O}_{5}$ ha $^{-1}$ as superphosphate) and potassium (100 $\mathrm{kg} \mathrm{K}_{2} \mathrm{O} \mathrm{ha}^{-1}$ as muriate of potash) were applied to all treatments. Zinc (5 $\mathrm{kg} \mathrm{Zn} \mathrm{ha}^{-1}$ as zinc sulfate) and boron (5 $\mathrm{kg} \mathrm{B} \mathrm{ha}^{-1}$ as calcite boronate) were also applied because a soil chemical analysis showed zinc and boron deficiency with values below $1 \mathrm{mg}$ $\mathrm{kg}^{-1}$ (Hirzel, 2007). All fertilizers were applied by hand broadcasting. Weeds were controlled at the initiation of tillering by applying Penoxsulam $\left(200 \mathrm{~g} \mathrm{~L}^{-1}\right)$ at $200 \mathrm{cc} \mathrm{ha}^{-1}$ and MCPA at $0.7 \mathrm{~L} \mathrm{ha}^{-1}$. The water level was kept at $10 \mathrm{~cm}$ above the soil surface until plant maturity.

\section{Crop measurements}

The following measurements were made in this field experiment: rice plants per $\mathrm{m}^{2}$, panicle number per $\mathrm{m}^{2}$, plant height and days to flowering. Plants per $\mathrm{m}^{2}$ and panicle number per $\mathrm{m}^{2}$ were sampled using a metal cylinder $\left(0.1 \mathrm{~m}^{2}\right)$ located in each plot, and separation was performed by hand-threshing. Plant height in centimeters and days to flowering were recorded for five individual plants in the middle of each sub-plot; this was considered representative of the rice-growing conditions. Mature samples were arranged ( 0.49 $\mathrm{m}^{2}$ ) and measured with a portable sensor (Satake Moistex, SS, Satake Corporation, Tokyo, Japan) in the center of each plot containing $23 \%$ moisture. Plants were air dried for two days, weighed on an electronic balance (Ohaus,GT-800, Ohaus Corporation, Ohio, USA) and finally processed in a 
stationary thresher with $15 \%$ rice grain moisture obtained using a sensor (Burrows, DMC- 700, Seedburo Equipment Company, Chicago, USA).

Grain yield $\left(\mathrm{t} \mathrm{ha}^{-1}\right)$ was determined by weighing the paddy rice using an electronic balance (Sartorius, 1216-MP, Sartorius GMBH, Göttingen, Germany). The 1000-grain weight was determined by weighing 1000 grains from each genotype in triplicate using an electronic balance (Sartorius, 1216-MP, Sartorius GMBH, Göttingen, Germany. The filled grain fraction was sieved in a $2.0 \mathrm{~mm}$ calibrator, and a $100 \mathrm{~g}$ sample was processed in a mill test (Suzuki, MT, Máquinas Suzuki S.A., Santa Cruz do Rio Pardo, Brazil). White grain rice was obtained by separating and filling the broken grains with a fraction separator (Satake, TRG, Satake Corporation, Tokyo, Japan). The harvest index (HI) was calculated by dividing the grain yield (paddy rice) by the biomass (grain + straw).

\section{Nitrogen agronomic efficiency (NAE)}

The NAE was calculated by dividing the total grain yield per nitrogen dose applied using the following relationship:

$\mathrm{NAE}=\left(\mathrm{Y}_{\mathrm{n}}-\mathrm{Y}_{0}\right) / \mathrm{N}$

where: NAE: nitrogen agronomic efficiency ( $\mathrm{kg}$ grain yield per $\mathrm{kg}^{-1} \mathrm{~N}$ applied); $\mathrm{Y}_{\mathrm{n}}$ : grain yield with $\mathrm{N}\left(\mathrm{kg} \mathrm{ha}^{-1}\right)$; $\mathrm{Y}_{0}$ : grain yield without $\mathrm{N}(\mathrm{kg}$ $\left.\mathrm{ha}^{-1}\right) ; \mathrm{N}$ : N dose $\left(\mathrm{kg} \mathrm{ha}^{-1}\right)$.

\section{Statistical analysis}

All data were exposed to an analysis of variance (ANOVA). Significant differences among treatments, genotypes and interactions were determined by a least significant difference test (LSD) at a $5 \%$ level of significance $(\mathrm{P} \leq 0.05)$ using the Statistical Analysis System (SAS Institute. 1998. UNIX Environment and derivatives. Version 6. SAS Institute Inc., Cary, North Carolina, USA).

\section{Results and discussion}

\section{Nitrogen dose}

Table 1 shows the variance analysis for the yield components, agronomic characteristics and NAE. The $\mathrm{N}$ dose had significant effects $(\mathrm{P} \leq 0.05)$ on the number of panicles per $\mathrm{m}^{2}$, NAE, grain yield, plant height, 1000-grain weight and the grain fill percentage. The $\mathrm{N}$ dose did not have significant effects on days to flowering, plants per $\mathrm{m}^{2}$ and the harvest index. Genotypes showed highly significant differences $(\mathrm{P} \leq 0.01)$ in all nitrogen parameters evaluated. The $\mathrm{N} \times$ genotype interaction $(\mathrm{N} \mathrm{x}$ $\mathrm{G})$ had significant effects only on plant height, 1000-grain weight and grain fill percentage. Similar results were obtained by Belder et al. (2004); the harvest index and biomass was not affected by $\mathrm{N}$ level.

\section{Genotype}

Rice genotypes had significant differences in plants per $\mathrm{m}^{2}$, panicles per $\mathrm{m}^{2}$, days to flowering, harvest index, NAE and grain yield (Table 2). The genotypes Quila 15690, Diamante, and Ámbar had a greater number of plants per $\mathrm{m}^{2}$, at 417,380

Table 1. Yield component variance analysis, agronomic characteristics and nitrogen agronomic efficiency in twenty rice genotypes.

\begin{tabular}{lcccc}
\hline Variable & $\mathrm{N}$ & $\mathrm{G}$ & $\mathrm{NxG}$ & $\mathrm{CV}(\%)$ \\
\hline Plants $\mathrm{m}^{-2}$ & $\mathrm{~ns}$ & $* *$ & $\mathrm{~ns}$ & 27.3 \\
Panicles $\mathrm{m}^{-2}$ & $* *$ & $* *$ & $\mathrm{~ns}$ & 13.1 \\
Days to flowering & $\mathrm{ns}$ & $* *$ & $\mathrm{~ns}$ & 2.6 \\
Harvest index & $\mathrm{ns}$ & $* *$ & $\mathrm{~ns}$ & 6.71 \\
NAE & $*$ & $* *$ & $\mathrm{~ns}$ & 40.7 \\
Grain yield & $* *$ & $* *$ & $\mathrm{~ns}$ & 15.4 \\
Plant height & $* *$ & $* *$ & $*$ & 5.22 \\
1000-grain weight & $*$ & $* *$ & $*$ & 4.1 \\
Grain fill & $* *$ & $* *$ & $*$ & 8.6 \\
\hline
\end{tabular}

(*): significant differences; $(* *)$ : highly significant difference; (ns): no significant difference;

$\mathrm{N}$ : nitrogen; G: genotype; CV: coefficient of variation. 
and 379 , respectively. The number of panicles is the greatest yield factor (Sun et al., 2012); in this study, the genotypes Ámbar, Quila 242105 and Quila 242101 had greater panicle numbers per $\mathrm{m}^{2}$, at 431, 428 and 422, respectively. For most genotypes, flowering occurred between 87 and 95 days after sowing. Quila 199604 and Quila 157302 had the shortest flowering times at 87 and 88 days, respectively.

Regarding the harvest index (HI), our results indicated that increases in cereal NAE can be obtained in varieties with high HI. The rice genotypes Ámbar, Quila 233006, and Quila 233202 had higher $\mathrm{HI}$ at $0.53 \mathrm{~kg}$ grain per $\mathrm{kg}$ total dry biomass. These HI measurements were greater than those obtained by Mahajan et al. (2012), which ranged from 0.35 to 0.42 . According to Inthapanya et al. (2000), this result suggests that cultivars with high $\mathrm{HI}$ are more efficient in nutrient use and perform well in different fertility conditions.

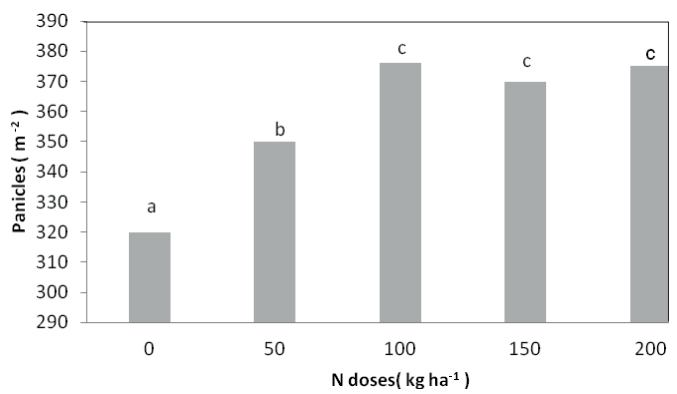

Figure 1. Panicle numbers from twenty rice genotypes with five doses of $\mathrm{N}$ in an Acuic Durixererts soil in Parral, Chile. Different letters indicate significant differences $(\mathrm{P} \leq 0.05)$ according to a LSD test. LSD: least significant difference.

The genotypes with the best grain yield were Brillante, Ámbar, Quila 242101 and Quila 242105 with yields of $11.2 ; 11.1 ; 10.7$ and $10.6 \mathrm{t} \mathrm{ha}^{-1}$, respectively. The rice genotypes Ámbar, Quila 233006, RQuila 17 and Quila 223202 obtained higher NAE measured at 47.7, 44.6, 43.8 and 42.6 $\mathrm{kg}_{\text {grain }} \mathrm{kg}^{-1} \mathrm{~N}$, respectively.

Table 2. Plants $\mathrm{m}^{-2}$, panicles $\mathrm{m}^{-2}$, days to flowering, harvest index (HI), nitrogen agronomic efficiency (NAE) and grain yield in twenty rice genotypes with five doses of $\mathrm{N}$ in an Acuic Durixerets soil in Parral, Chile.

\begin{tabular}{|c|c|c|c|c|c|c|}
\hline Genotype & Plants $\mathrm{m}^{-2}$ & Panicles $m^{-2}$ & $\begin{array}{l}\text { Days to } \\
\text { flowering }\end{array}$ & HI & $\begin{array}{c}\mathrm{NAE} \\
\left(\mathrm{kg} \text { grain } \mathrm{kg}^{-1} \mathrm{~N}\right)\end{array}$ & $\begin{array}{l}\text { Grain yield } \\
\left(\mathrm{t} \mathrm{ha}^{-1}\right)\end{array}$ \\
\hline Ámbar & $379.0 \mathrm{ab}^{1}$ & $431.0 \mathrm{a}$ & $92.0 \mathrm{de}$ & $0.53 \mathrm{ab}^{*}$ & $47.7 \mathrm{a}$ & $11.1 \mathrm{ab}$ \\
\hline Quila 233006 & $269.0 \mathrm{~d}$ & $350.0 \mathrm{gh}$ & 92.0 cde & $0.53 \mathrm{a}$ & $44.6 \mathrm{ab}$ & $10.0 \mathrm{cdef}$ \\
\hline RQuila 17 & $324.0 \mathrm{bcd}$ & $351.0 \mathrm{gh}$ & $91.0 \mathrm{e}$ & $0.49 \mathrm{def}$ & $43.8 \mathrm{abc}$ & $9.9 \mathrm{cdef}$ \\
\hline Quila 223202 & $363.0 \mathrm{abc}$ & $398.0 \mathrm{bcd}$ & $89.0 \mathrm{f}$ & $0.53 \mathrm{ab}$ & $42.6 \mathrm{abc}$ & 10.4 abcde \\
\hline Quila 121304 & $314.0 \mathrm{~cd}$ & 375.0 defg & $93.0 \mathrm{bcd}$ & $0.51 \mathrm{bcd}$ & $41.7 \mathrm{abcd}$ & 10.2 bcde \\
\hline RQuila 28 & $376.0 \mathrm{ab}$ & $396.0 \mathrm{~cd}$ & $93.0 \mathrm{bcd}$ & $0.48 \mathrm{efg}$ & 41.5 abcde & $9.1 \mathrm{fg}$ \\
\hline CINIA 857 & $354.0 \mathrm{bc}$ & $387.0 \mathrm{de}$ & $92.0 \mathrm{de}$ & 0.50 cde & 41.5 abcde & 10.4 abcde \\
\hline Cuarzo & $356.0 \mathrm{bc}$ & 375.0 defg & $93.0 \mathrm{bcd}$ & $0.52 \mathrm{abc}$ & 40.6 abcde & 10.1 bcde \\
\hline Quila 242105 & $326.0 \mathrm{bcd}$ & $428.0 \mathrm{ab}$ & $93.0 \mathrm{bcd}$ & $0.52 \mathrm{abc}$ & 39.8 abcdef & $10.6 \mathrm{abcd}$ \\
\hline Quila 242101 & $351.0 \mathrm{bc}$ & $422.0 \mathrm{abc}$ & $95.0 \mathrm{a}$ & $0.52 \mathrm{abc}$ & 38.0 abcdefg & $10.7 \mathrm{abc}$ \\
\hline Quila 201704 & $349.0 \mathrm{bc}$ & $364.0 \mathrm{efg}$ & $89.0 \mathrm{f}$ & $0.46 \mathrm{gh}$ & 37.2 abcdefg & $8.8 \mathrm{gh}$ \\
\hline Diamante & $380.0 \mathrm{ab}$ & $349.0 \mathrm{gh}$ & $94.0 \mathrm{abc}$ & $0.47 \mathrm{fgh}$ & 37.0 bcdefg & $9.9 \mathrm{cdef}$ \\
\hline Brillante & $325.0 \mathrm{bcd}$ & $331.0 \mathrm{hi}$ & $93.0 \mathrm{bcd}$ & $0.53 \mathrm{a}$ & 36.0 bcdefg & $11.2 \mathrm{a}$ \\
\hline Zafiro & $306.0 \mathrm{~cd}$ & $353.0 \mathrm{fgh}$ & $93.0 \mathrm{bcd}$ & $0.50 \mathrm{~cd}$ & 34.0 bcdefg & $9.8 \mathrm{cdef}$ \\
\hline Quila 157302 & $351.0 \mathrm{bc}$ & $356.0 \mathrm{fgh}$ & $88.0 \mathrm{fg}$ & $0.46 \mathrm{hi}$ & $33.6 \mathrm{cdefg}$ & 7.9 ih \\
\hline Quila 156906 & $417.0 \mathrm{a}$ & $363.0 \mathrm{efg}$ & $89.0 \mathrm{fg}$ & $0.49 \mathrm{def}$ & $31.2 \mathrm{defg}$ & $9.6 \mathrm{fg}$ \\
\hline Quila 242415 & $353.0 \mathrm{bc}$ & 370.0 defg & $89.0 \mathrm{fg}$ & $0.46 \mathrm{gh}$ & 30.9 efg & 8.0 ih \\
\hline Quila 199604 & $322.0 \mathrm{bcd}$ & $318.0 \mathrm{i}$ & $87.0 \mathrm{~g}$ & $0.44 \mathrm{i}$ & 30.9 efg & $7.4 \mathrm{i}$ \\
\hline Quila 233008 & $315.0 \mathrm{~cd}$ & $382.0 \mathrm{def}$ & $94.0 \mathrm{ab}$ & $0.52 \mathrm{abc}$ & $29.5 \mathrm{fg}$ & $9.7 \mathrm{def}$ \\
\hline Dmu $81 \mathrm{~m}$ & $324.0 \mathrm{bcd}$ & 370.0 defg & 93.0 bcde & $0.49 \mathrm{def}$ & $29.2 \mathrm{~g}$ & $9.1 \mathrm{fg}$ \\
\hline LSD G & 58.2 & 30.4 & 1.0 & 0.02 & 10.7 & 0.9 \\
\hline
\end{tabular}

${ }^{1}$ Means with different letters in columns indicate significant differences $(\mathrm{P} \leq 0.05)$ according to a LSD test; LSD: least significant difference; HI: harvest index; N: nitrogen. 
$\mathrm{N}$ dose had a significant effect on the number of panicles per $\mathrm{m}^{2}$ up to $100 \mathrm{~kg} \mathrm{~N} \mathrm{ha}^{-1}$; at 150 and $200 \mathrm{~kg} \mathrm{~N} \mathrm{ha}^{-1}$; however, no significant difference in panicle number was observed (Figure 1). According to Bond et al. (2008), this result indicates that rice breeding has decreased the nitrogen load in soils with low natural fertility. Hirzel (2007) suggested that this is because of the second application of $\mathrm{N}$ at the tillering initiation stage stimulates panicle production.

\section{Nitrogen $(N) \times$ genotype $(G)$ interaction}

The interaction of $\mathrm{N} \mathrm{x}$ G had significant effects only on the plant height, 1000-grain weight and grain fill percentage. All genotypes had an increase in plant height (Table 3) as the $\mathrm{N}$ dose increased; each genotype had a unique response. The greatest significant differences in plant height were obtained between 0 and $50 \mathrm{~kg} \mathrm{~N} \mathrm{ha}^{-1}$. For most genotypes, plant height values ranged from 2 and $11 \mathrm{~cm}$. In the genotypes Quila 201704, Quila 233008 and RQuila 28 genotypes, the plant height values were below the average.

Genotypes showed differential responses in plant height values. Diamante, Quila 201704, Brillante, and Dmu $8 \mathrm{~m}$ had increases in plant height at $\mathrm{N}$ doses greater than $100 \mathrm{~kg} \mathrm{~N} h a^{-1}$. Quila 233008 and Quila 223202 did not show significant increases in plant height at $\mathrm{N}$ doses greater than $100 \mathrm{~kg}$ $\mathrm{N} \mathrm{ha}^{-1}$. This varietal characteristic is important in determining grain yield; short cultivars had greater grain yields $\left(7.5 \mathrm{t} \mathrm{ha}^{-1}\right)$ than tall cultivars $\left(6.7 \mathrm{t} \mathrm{ha}^{-1}\right)$ and avoid lodging due to heavy rain and wind (Koutroubas and Ntanos, 2003; Timsina et al., 2001)

The 1000-grain weight of the twenty rice genotypes at the five $\mathrm{N}$ doses is summarized in Table 3. The results are similar to those for cultivars used in Chile (27-33 g), (Alvarado and Hernaíz, 2007a). The 1000-grain weight for

Table 3. Plant height, 1000-grain weight and grain fill percentage in twenty rice genotypes with five doses of $\mathrm{N}$ in an Acuic Durixererts soil in Parral, Chile.

\begin{tabular}{|c|c|c|c|c|c|c|c|c|c|c|c|c|c|c|c|}
\hline \multirow[b]{2}{*}{ Genotype } & \multicolumn{5}{|c|}{ Plant height $(\mathrm{cm})$} & \multicolumn{5}{|c|}{ 1000-grain weight (g) } & \multicolumn{5}{|c|}{ Grain fill percentage (\%) } \\
\hline & $\mathrm{N}_{\mathrm{o}}$ & $\mathrm{N}_{50}$ & $\mathrm{~N}_{100}$ & $\mathrm{~N}_{150}$ & $\mathrm{~N}_{200}$ & $\mathrm{~N}_{\mathrm{o}}$ & $\mathrm{N}_{50}$ & $\mathrm{~N}_{100}$ & $\mathrm{~N}_{150}$ & $\mathrm{~N}_{200}$ & $\mathrm{~N}_{\mathrm{o}}$ & $\mathrm{N}_{50}$ & $\mathrm{~N}_{100}$ & $\mathrm{~N}_{150}$ & $\mathrm{~N}_{200}$ \\
\hline Quila 199604 & $59.5 \mathrm{~d}^{1}$ & $65.5 \mathrm{c}$ & $68.0 \mathrm{abc}$ & $69.3 \mathrm{a}$ & $68.5 \mathrm{ab}$ & $37.1 \mathrm{a}$ & $34.0 \mathrm{c}$ & $38.0 \mathrm{a}$ & $37.2 \mathrm{ab}$ & $36.9 \mathrm{~b}$ & $28.9 \mathrm{~g}$ & $37.5 \mathrm{j}$ & $39.5 \mathrm{i}$ & $40.2 \mathrm{j}$ & $47.5 \mathrm{~h}$ \\
\hline RQuila 17 & $64.8 \mathrm{c}$ & $68.8 b$ & $74.0 \mathrm{a}$ & $76.3 \mathrm{a}$ & $75.8 \mathrm{a}$ & $37.4 \mathrm{a}$ & $36.8 \mathrm{~b}$ & $36.1 \mathrm{~b}$ & $34.7 \mathrm{c}$ & $33.5 \mathrm{~d}$ & $53.2 \mathrm{cde}$ & $56.1 \mathrm{bcd}$ & de $64.2 \mathrm{a}$ & $65.7 \mathrm{a}$ & $62.7 \mathrm{abc}$ \\
\hline Diamante & $64.3 \mathrm{~d}$ & $67.0 \mathrm{c}$ & $72.0 \mathrm{~b}$ & $78.8 \mathrm{a}$ & $79.5 \mathrm{a}$ & $36.8 \mathrm{a}$ & $36.7 \mathrm{a}$ & $35.3 b$ & $34.3 b$ & $34.5 \mathrm{~b}$ & $55.4 \mathrm{bc}$ & $58.6 \mathrm{~b}$ & $63.5 \mathrm{ab}$ & $65.4 \mathrm{ab}$ & $65.5 \mathrm{a}$ \\
\hline Quila 121304 & $59.3 \mathrm{~d}$ & $68.0 \mathrm{c}$ & $71.0 \mathrm{~b}$ & $68.5 \mathrm{bc}$ & $73.8 \mathrm{a}$ & $37.1 \mathrm{a}$ & $35.2 \mathrm{bc}$ & $34.6 \mathrm{c}$ & $34.3 \mathrm{c}$ & $35.7 \mathrm{~b}$ & $55.4 \mathrm{bc}$ & $58.4 \mathrm{~b} \quad 6$ & $63.3 \mathrm{abc}$ & 63.6abcd & $63.7 \mathrm{ab}$ \\
\hline Zafiro & $59.5 \mathrm{~d}$ & $63.3 \mathrm{c}$ & $69.3 b$ & $73.3 \mathrm{a}$ & $73.8 \mathrm{a}$ & $35.9 \mathrm{a}$ & $36.1 \mathrm{a}$ & $35.2 \mathrm{ab}$ & $34.4 \mathrm{bc}$ & $33.4 \mathrm{c}$ & $58.6 \mathrm{~b}$ & $57.4 \mathrm{bc}$ & $62.2 \mathrm{abc}$ & $65.2 \mathrm{ab}$ & $64.5 \mathrm{a}$ \\
\hline Dmu $81 \mathrm{~m}$ & $57.8 \mathrm{~d}$ & $67.3 \mathrm{c}$ & $69.5 \mathrm{bc}$ & $71.5 \mathrm{~b}$ & $75.3 \mathrm{a}$ & $35.8 \mathrm{a} \quad 3$ & $35.3 \mathrm{ab}$ & $34.6 \mathrm{bc}$ & $34.2 \mathrm{c}$ & $34.3 \mathrm{bc}$ & $54.2 \mathrm{~cd}$ & $58.2 \mathrm{~b}$ & $61.1 \mathrm{abc}$ & 64.1abcd & $64.8 \mathrm{a}$ \\
\hline Cuarzo & $59.5 \mathrm{~d}$ & $62.8 \mathrm{c}$ & $70.5 b$ & $70.5 b$ & $73.8 \mathrm{a}$ & $36.4 \mathrm{a}$ & $35.1 \mathrm{~b}$ & $35.2 \mathrm{~b}$ & $34.2 b$ & $32.9 \mathrm{c}$ & $51.3 \mathrm{de}$ & $56.7 \mathrm{bcd}$ & $59.7 \mathrm{~cd}$ & 60.9 fde & $62.3 \mathrm{abcd}$ \\
\hline Brillante & $57.8 \mathrm{c}$ & $67.3 b$ & $69.5 \mathrm{~b}$ & $75.0 \mathrm{a}$ & $75.5 \mathrm{a}$ & $35.6 \mathrm{a}$ & $35.6 \mathrm{a}$ & $34.8 \mathrm{a}$ & $33.3 b$ & $32.3 b$ & $55.7 \mathrm{bc}$ & $56.6 \mathrm{bcde}$ & $60.6 b c$ & $62.1 \mathrm{bcde}$ & $63.2 \mathrm{abc}$ \\
\hline CINIA 857 & $60.3 \mathrm{c}$ & $66.5 b$ & $70.5 \mathrm{a}$ & $71.3 \mathrm{a}$ & $72.0 \mathrm{a}$ & $34.4 \mathrm{ab}$ & $34.7 \mathrm{a}$ & $34.8 \mathrm{a}$ & $33.6 \mathrm{bc}$ & $32.9 \mathrm{c}$ & $52.4 \mathrm{cde}$ & $53.7 \mathrm{def}$ & $60.5 b c$ & $64.5 \mathrm{abc}$ & $60.0 \mathrm{fcde}$ \\
\hline Quila 233006 & $61.0 \mathrm{c}$ & $66.5 b$ & $68.0 \mathrm{ab}$ & $69.8 \mathrm{a}$ & $69.0 \mathrm{ab}$ & $33.2 \mathrm{ab}$ & $33.6 \mathrm{a}$ & $33.8 \mathrm{a}$ & $33.2 \mathrm{ab}$ & $32.3 b$ & $43.7 f$ & $45.9 \mathrm{~h}$ & $50.4 \mathrm{~g}$ & $58.2 \mathrm{fg}$ & $59.1 \mathrm{fde}$ \\
\hline Quila 223202 & $63.3 \mathrm{c}$ & $74.5 \mathrm{a}$ & $75.0 \mathrm{a}$ & $71.5 \mathrm{~b}$ & $76.0 \mathrm{a}$ & $30.6 \mathrm{a}$ & $30.8 \mathrm{a}$ & $30.7 \mathrm{a}$ & $31.5 \mathrm{a}$ & $30.6 \mathrm{a}$ & $51.8 \mathrm{de}$ & $49.2 \mathrm{hg}$ & $50.5 \mathrm{~g}$ & $53.2 \mathrm{i}$ & $48.9 \mathrm{~h}$ \\
\hline Quila 242105 & $57.0 \mathrm{~b}$ & $62.3 \mathrm{a}$ & $62.8 \mathrm{a}$ & $63.3 \mathrm{a}$ & $64.3 \mathrm{a}$ & $30.8 \mathrm{ab}$ & $31.6 \mathrm{a}$ & $30.4 \mathrm{~b}$ & $30.5 b$ & $30.2 b$ & $53.1 \mathrm{cde}$ & $57.9 \mathrm{bc}$ & $57.1 \mathrm{de}$ & $57.1 \mathrm{gh}$ & $57.5 \mathrm{fg}$ \\
\hline Quila 242101 & $55.5 \mathrm{c}$ & $58.3 b$ & $64.3 \mathrm{a}$ & $59.8 \mathrm{~b}$ & $62.5 \mathrm{a}$ & $31.3 \mathrm{a}$ & $31.3 \mathrm{a}$ & $30.8 \mathrm{a}$ & $30.5 \mathrm{a}$ & $28.9 \mathrm{~b}$ & $52.5 \mathrm{cde}$ & $58.4 \mathrm{~b}$ & $54.5 \mathrm{fe}$ & $60.2 \mathrm{fge}$ & $49.6 \mathrm{~h}$ \\
\hline Quila 233008 & $63.8 \mathrm{~d}$ & $64.8 \mathrm{~cd}$ & $68.5 \mathrm{bc}$ & $65.5 b c$ & $69.8 \mathrm{ab}$ & $30.5 \mathrm{ab}$ & $29.8 \mathrm{bc}$ & $30.8 \mathrm{a}$ & $29.6 \mathrm{bc}$ & $29.4 \mathrm{c}$ & $51.8 \mathrm{de}$ & $53.2 \mathrm{ef}$ & $51.7 \mathrm{fg}$ & $54.2 \mathrm{hi}$ & $57.7 \mathrm{fg}$ \\
\hline Quila 201704 & $68.0 \mathrm{~d}$ & $69.3 \mathrm{~cd}$ & $71.5 \mathrm{bc}$ & $72.3 \mathrm{a}$ & $75.5 \mathrm{a}$ & $30.1 \mathrm{ab}$ & $30.5 \mathrm{a}$ & $29.4 b$ & $29.2 \mathrm{~b}$ & $30.5 \mathrm{a}$ & $52.7 \mathrm{cde}$ & $51.8 \mathrm{fg}$ & $55.9 \mathrm{e}$ & $59.2 \mathrm{fge}$ & $55.2 \mathrm{~g}$ \\
\hline Quila 157302 & $53.0 \mathrm{c}$ & $65.8 \mathrm{ab}$ & $69.3 \mathrm{a}$ & $69.8 \mathrm{a}$ & $69.3 \mathrm{a}$ & $30.2 \mathrm{a}$ & $28.8 \mathrm{~b}$ & $29.1 \mathrm{~b}$ & $28.8 \mathrm{~b}$ & $29.3 \mathrm{ab}$ & $40.1 \mathrm{f}$ & $42.1 \mathrm{i}$ & $46.7 \mathrm{~h}$ & $52.0 \mathrm{i}$ & $48.7 \mathrm{~h}$ \\
\hline Quila 156906 & $59.8 \mathrm{~d}$ & $67.3 \mathrm{c}$ & $72.5 \mathrm{a}$ & $72.0 \mathrm{ab}$ & $69.8 \mathrm{bc}$ & $28.3 \mathrm{abc}$ & $29.3 a$ & $29.0 \mathrm{ab}$ & $27.8 \mathrm{c}$ & $28.0 \mathrm{bc}$ & $40.9 f$ & $40.0 \mathrm{ij}$ & $50.0 \mathrm{gh}$ & $52.5 \mathrm{i}$ & $55.2 \mathrm{~g}$ \\
\hline RQuila 28 & $56.3 \mathrm{~b}$ & $58.8 \mathrm{~b}$ & $68.0 \mathrm{a}$ & $66.5 \mathrm{a}$ & $66.3 \mathrm{a}$ & $30.4 \mathrm{a}$ & $29.2 b$ & $27.9 \mathrm{c}$ & $26.9 \mathrm{c}$ & $27.4 \mathrm{c}$ & $62.6 \mathrm{a}$ & $63.2 \mathrm{a}$ & $63.8 \mathrm{ab}$ & $65.5 \mathrm{a}$ & $65.4 \mathrm{a}$ \\
\hline Ámbar & $54.0 \mathrm{~d}$ & d $59.0 \mathrm{c}$ & $60.5 \mathrm{bc}$ & $63.3 \mathrm{a}$ & $62.3 \mathrm{ab}$ & $27.6 \mathrm{bc}$ & $27.8 \mathrm{ab}$ & $28.7 \mathrm{a}$ & $27.7 \mathrm{abc}$ & $26.8 \mathrm{c}$ & $50.2 \mathrm{de}$ & $56.8 \mathrm{bcd}$ & $62.4 \mathrm{abc}$ & $61.6 \mathrm{cde}$ & $60.8 \mathrm{bcde}$ \\
\hline Quila 242415 & $57.0 \mathrm{~b}$ & $62.3 \mathrm{a}$ & $62.8 \mathrm{a}$ & $63.3 \mathrm{a}$ & $62.3 \mathrm{ab}$ & $27.3 \mathrm{ab}$ & $27.3 \mathrm{a}$ & $26.3 b$ & $26.3 \mathrm{ab}$ & $25.8 \mathrm{~b}$ & $54.3 \mathrm{~cd}$ & $54.8 \mathrm{cdef}$ & f $57.0 \mathrm{de}$ & $54.1 \mathrm{hi}$ & $57.9 \mathrm{fge}$ \\
\hline LSD N x G & & & 2.46 & & & & & 1.01 & & & & & 3.4 & & \\
\hline
\end{tabular}

${ }^{1}$ Means with different letters in rows indicate significant differences $(\mathrm{P} \leq 0.05)$ according to a LSD test; LSD: least significant difference; $\mathrm{N}_{\mathrm{o}}$ : nitrogen dose. 
Quila 223202 did not change with increasing $\mathrm{N}$ doses. The 1000-grain weight for RQuila 17 declined significantly with increasing $\mathrm{N}$ doses. The 1000-grain weight decreased with increasing $\mathrm{N}$ doses of up to $100 \mathrm{~kg} \mathrm{~N}^{-1}$ in Diamante and up to $150 \mathrm{~kg} \mathrm{~N} \mathrm{ha}^{-1}$ in Brillante. The value of this parameter ranged from 25.8 to $38.0 \mathrm{~g}$ but decreased when $\mathrm{N}$ doses higher than $100 \mathrm{~kg} \mathrm{~N}$ $\mathrm{ha}^{-1}$ were used. These results could be attributed to an increase total number of grains per panicle, a decrease in panicle length and lighter grains (Koutroubas and Ntanos, 2003; Sun et al., 2012). The results support those of Kato et al. (2009), who obtained a 1000 -grain weight between 24.7 to $35.6 \mathrm{~g}$ when applying $180 \mathrm{~kg} \mathrm{~N} \mathrm{ha}^{-1}$ in a Typic Fluvaquent soil.

$\mathrm{N}$ had a significant effect of on the grain fill percentage in the genotype RQuila 28 compared to the other genotypes evaluated. RQuila 28 had 62.6 and $63.2 \%$ grain fill at 0 and $50 \mathrm{~kg}$ of $\mathrm{N} \mathrm{ha}$ ${ }^{1}$, respectively (Table 3). At a dose of $100 \mathrm{~kg} \mathrm{~N}$ ha $^{-1}$, RQuila 17 had a higher grain fill percentage (64.2\%). Grain fill was not significantly different in the following genotypes: Rquila 28, Diamante, Quila 121304, Ámbar, Zafiro and Dmu 81m.

At the $150 \mathrm{~kg} \mathrm{~N}^{-1}$ dose, RQuila 17 had a greater grain fill percentage (65.7\%). Grain fill was not significantly different in the genotypes RQuila 28, Diamante, Zafiro, CINIA 857, Dmu 81m and Quila 121304 at this dose. Diamante had the highest grain fill percentage (65.5\%) at a dose of $200 \mathrm{~kg}$ of $\mathrm{N} \mathrm{ha}^{-1}$; however, grain fill was not significantly different in RQuila 28, Dmu 81m, Zafiro, Quila 121304, Brillante, RQuila 17 and Cuarzo.

In this study, the percentage of grain fill ranged from $28.9 \%$ in Quila 199604 at $0 \mathrm{~kg} \mathrm{~N} \mathrm{ha}^{-1}$ to $65.7 \%$ in RQuila 17 at $150 \mathrm{~kg} \mathrm{~N}^{-1}$. This result illustrates the genotypic differences observed. At a low dose of N, only the R Quila 28, CINIA 857 , Brillante, RQuila17 genotypes had up to $60 \%$ grain fill and plant characteristics that were desirable in selected genotypes with higher NAE. In general, the percentage of grain fill decreased at $\mathrm{N}$ doses higher than $100 \mathrm{~kg} \mathrm{~N} \mathrm{ha}^{-1}$. This result was most likely due to the excessively absorbed $\mathrm{N}$ being allocated to straw over grain (Jian-feng et al., 2011).

$\mathrm{N}$ fertilization had a positive effect on the grain fill percentage. A genotype analysis revealed that only RQuila 28 had grain fill greater than $60 \%$ at 0 and $50 \mathrm{~kg} \mathrm{~N} \mathrm{ha}^{-1}$. According to Alvarado and Hernaíz (2007b), these differences are not only caused by nitrogen but also by other factors, such as the cultivar, the climate conditions between flowering and harvest, the harvesting system and grain moisture.

\section{Grain yield and NAE}

Interaction effects between $\mathrm{G}$ and $\mathrm{N}$ were not significant for grain yield and NAE. Similar results were obtained by Singh et al. (1998). Grain yield and NAE for twenty rice genotypes and five $\mathrm{N}$ doses are summarized in Table 4. Grain yield was positively correlated with $\mathrm{N}$ dose, except between 100 and $150 \mathrm{~kg} \mathrm{~N}^{-1}$ (Figure 2). Similar results were reported by Poshtmasari et al. (2007), who found significant grain yield increases in genotypes at a $200 \mathrm{~kg} \mathrm{~N} \mathrm{ha}^{-1}$ dose. In contrast, Singh et al. (1998) found no significant increase in grain yield between 150 to $200 \mathrm{~kg} \mathrm{~N} \mathrm{ha}^{-1}$. Lodging occurred in some genotypes, and spikelet sterility was high in the aforementioned study. Mahajan et al. (2012) reported that the application of more than $120 \mathrm{~kg} \mathrm{~N} \mathrm{ha}^{-1}$ did not show any advantage in terms of $\mathrm{N}$ use and yield.

NAE decreased when $\mathrm{N}$ application was between 50 and $200 \mathrm{~kg} \mathrm{~N}^{-1}$ (Figure 3). This is in agreement with results from Ohnishi et al. (1999) and Peng et al. (2007). The lower NAE in rice at 150 and $200 \mathrm{~kg} \mathrm{~N} \mathrm{ha}^{-1}$ suggests that there are no yield benefits from additional application of $\mathrm{N}$ above $100 \mathrm{~kg} \mathrm{ha}^{-1}$ (Timsina et al., 2001). This result indicates that in genotypes with differences in NAE, the changes are due to biochemical and physiological characteristics, root uptake, re- 
Table 4. Rice grain yield and nitrogen agronomic efficiency (NAE) in twenty rice genotypes with five doses of $\mathrm{N}$ in an Acuic Durixererts soil in Parral, Chile.

\begin{tabular}{|c|c|c|c|c|c|c|c|c|c|}
\hline \multirow[b]{2}{*}{ Genotype } & \multicolumn{5}{|c|}{$\begin{array}{l}\text { Grain yield } \\
\left(\mathrm{t} \mathrm{ha}^{-1}\right)\end{array}$} & \multicolumn{4}{|c|}{$\begin{array}{c}\mathrm{NAE} \\
\left(\mathrm{kg}_{\left.\text {grain } \mathrm{kg}^{-1} \mathrm{~N}\right)}\right.\end{array}$} \\
\hline & $\mathrm{N}_{\mathrm{o}}$ & $\mathrm{N}_{50}$ & $\mathrm{~N}_{100}$ & $\mathrm{~N}_{150}$ & $\mathrm{~N}_{200}$ & $\mathrm{~N}_{50}$ & $\mathrm{~N}_{100}$ & $\mathrm{~N}_{150}$ & $\mathrm{~N}_{200}$ \\
\hline Quila 199604 & 5.4 & 8.1 & 8.3 & 10.4 & 9.7 & 48.56 & 26.14 & 29.76 & 19.17 \\
\hline RQuila 17 & 6.6 & 9.5 & 12.7 & 12.1 & 14.9 & 51.16 & 54.58 & 32.78 & 36.80 \\
\hline Diamante & 7.2 & 9.5 & 12.0 & 12.5 & 14.3 & 41.20 & 43.25 & 31.73 & 31.84 \\
\hline Quila 121304 & 7.0 & 9.4 & 13.1 & 13.8 & 14.0 & 42.86 & 53.41 & 39.92 & 30.75 \\
\hline Zafiro & 6.4 & 7.8 & 10.1 & 12.4 & 12.4 & 29.02 & 37.13 & 39.73 & 30.11 \\
\hline Dmu $81 \mathrm{~m}$ & 7.5 & 9.7 & 11.7 & 11.7 & 10.8 & 39.80 & 37.27 & 24.83 & 14.75 \\
\hline Cuarzo & 6.5 & 9.4 & 11.5 & 9.9 & 13.4 & 56.20 & 49.43 & 22.66 & 34.08 \\
\hline Brillante & 8.8 & 11.1 & 13.6 & 13.6 & 16.1 & 40.90 & 42.76 & 28.25 & 32.24 \\
\hline CINIA 857 & 7.7 & 11.3 & 12.5 & 13.4 & 13.4 & 64.29 & 42.66 & 33.79 & 25.17 \\
\hline Quila 233006 & 6.9 & 10.5 & 12.2 & 12.7 & 14.9 & 63.42 & 46.64 & 33.64 & 34.85 \\
\hline Quila 223202 & 7.6 & 11.1 & 12.2 & 12.7 & 14.9 & 63.81 & 41.31 & 29.69 & 35.64 \\
\hline Quila 242105 & 8.1 & 10.6 & 14.6 & 14.1 & 13.5 & 43.81 & 56.87 & 39.48 & 23.64 \\
\hline Quila 242101 & 8.1 & 10.5 & 12.7 & 14.7 & 15.2 & 42.21 & 40.42 & 38.04 & 31.44 \\
\hline Quila 233008 & 8.0 & 9.8 & 12.4 & 12.5 & 13.1 & 31.39 & 38.10 & 26.36 & 22.24 \\
\hline Quila 201704 & 6.1 & 8.7 & 10.2 & 13.0 & 11.4 & 46.53 & 36.99 & 41.31 & 24.05 \\
\hline Quila 157302 & 5.6 & 8.4 & 10.3 & 6.9 & 11.1 & 48.62 & 40.17 & 21.75 & 23.93 \\
\hline Quila 156906 & 9.4 & 9.0 & 11.9 & 12.2 & 13.6 & 28.37 & 40.53 & 28.43 & 27.53 \\
\hline RQuila 28 & 6.5 & 10.1 & 12.1 & 11.5 & 11.2 & 64.19 & 50.28 & 30.32 & 21.21 \\
\hline $\begin{array}{l}\text { Ámbar } \\
\text { Media } \\
\text { LSD N x G }\end{array}$ & $\begin{array}{c}7.2 \\
6.3 \mathrm{a}^{1} \\
0.91\end{array}$ & $\begin{array}{c}9.6 \\
8.6 \mathrm{~b}\end{array}$ & $\begin{array}{c}14.6 \\
10.7 \mathrm{c}\end{array}$ & $\begin{array}{c}14.0 \\
11.0 \mathrm{c}\end{array}$ & $\begin{array}{c}17.5 \\
11.9 \mathrm{~d}\end{array}$ & $\begin{array}{c}41.69 \\
46.27 \mathrm{a} \\
11.84\end{array}$ & $\begin{array}{c}64.46 \\
44.02 \mathrm{~b}\end{array}$ & $\begin{array}{c}39.70 \\
31.72 \mathrm{c}\end{array}$ & $\begin{array}{r}45.09 \\
28.30 \mathrm{c}\end{array}$ \\
\hline
\end{tabular}

${ }^{1}$ Means with different letters in the rows indicate significant differences ( $\left.\mathrm{P} \leq 0.05\right)$ according to a LSD test; LSD: least significant difference; $\mathrm{N}_{\mathrm{o}}$ : nitrogen dose.

mobilization and translocation of absorbed $\mathrm{N}$ to different plant organs (Ladha et al., 1998), plant density, micronutrient deficiency (Hossain et al., 2005) and soil type.

Table 4 shows that the NAE of the twenty rice genotypes varied between 14.75 and $64.46 \mathrm{~kg}$ grain $\mathrm{kg}^{-1} \mathrm{~N}$. These results are in agreement with the those reported by Samonte et al. (2006), who reported NAE between 25.3 and $63.9 \mathrm{~kg}_{\text {grain }} \mathrm{kg}^{-1} \mathrm{~N}$. According to Fageria and Barbosa (2001) and Ahmad et al. (2008), the genotypes that produce high grain yields in a lower $\mathrm{N}$ and respond well to added $\mathrm{N}$ are the most desirable; these genotypes can be grown with a limited $\mathrm{N}$ supply for environmentally friendly farming systems. The rice genotypes Ámbar, Quila 233006, Quila 223202, CINIA 857 and RQuila 17 had greater NAE values at lower $\mathrm{N}$ doses.

In this study, the genotypes were selected ac- cording to NAE, 1000-grain weight and grain fill percentage. The best rice genotypes were as follows: Quila 233006, Quila 223202, CINIA 857 and RQuila28 at a dose of $50 \mathrm{~kg} \mathrm{~N} \mathrm{ha}^{-1}$ and Ámbar Quila 17 and Quila 242105 at a dose of $100 \mathrm{~kg} \mathrm{~N} \mathrm{ha}^{-1}$. These genotypes had a NAE average of $64 \mathrm{~kg}$ grain $\mathrm{kg}^{-1} \mathrm{~N}$, a 1000 -grain weight of $32 \mathrm{~g}$ and $53 \%$ grain fill at $50 \mathrm{~kg} \mathrm{~N} \mathrm{ha}^{-1}$; RQuila 28 had a grain fill of $63.2 \%$. At the $100 \mathrm{~kg} \mathrm{~N}$ ha $^{-1}$ dose, the best genotypes had a NAE value of $59 \mathrm{~kg}$ grain $\mathrm{kg}^{-1} \mathrm{~N}$, a 1000 -grain weight of 32 $\mathrm{g}$ and a $61 \%$ grain fill. Quila 223202 had high

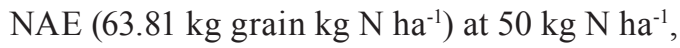
but not selected due to a low grain fill percentage (49.2\%). In general, these rice genotypes revealed yield components that may be of interest when developing new cultivars. Samonte et al. (2006) reported that a rice genotype with a higher $\mathrm{N}$ translocation ratio would be desirable if it was capable of remobilizing $\mathrm{N}$ into the grain during 


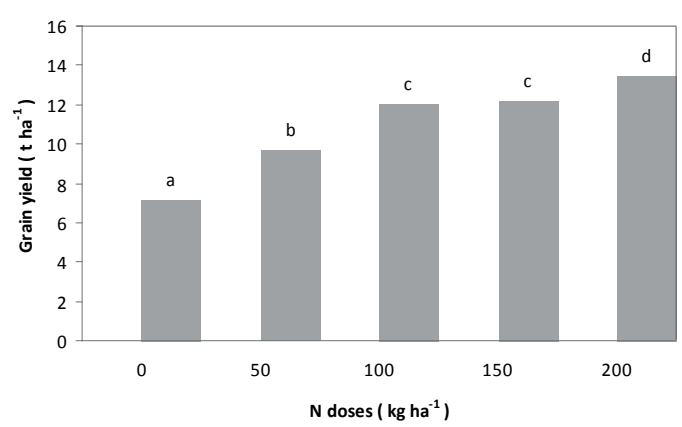

Figure 2. Grain yield of twenty rice genotypes with five doses of $\mathrm{N}$ in an Acuic Durixererts soil in Parral, Chile. Different letters indicate significant differences $(\mathrm{P} \leq 0.05)$ according to a LSD test. LSD: least significant difference.

grain fill.

This study demonstrated that the application of $\mathrm{N}$ improved grain yield, panicle number and grain fill percentage but had negative effects on plant height,1000-grain weight and NAE. The genotypes selected had greater NAE at doses of 50 and $100 \mathrm{~kg} \mathrm{~N} \mathrm{ha}^{-1}$. The interaction between nitrogen $\mathrm{x}$ genotype had a significant effect only on plant height, 1000-grain weight and grain fill percentage. Among the genotypes evaluated, Quila 233006, Quila 223202, CINIA 857 and RQuila17 at $50 \mathrm{~kg} \mathrm{~N} \mathrm{ha}^{-1}$ and Ámbar, RQuila 17 and Quila 242105 at $100 \mathrm{~kg} \mathrm{~N} \mathrm{ha}^{-1}$

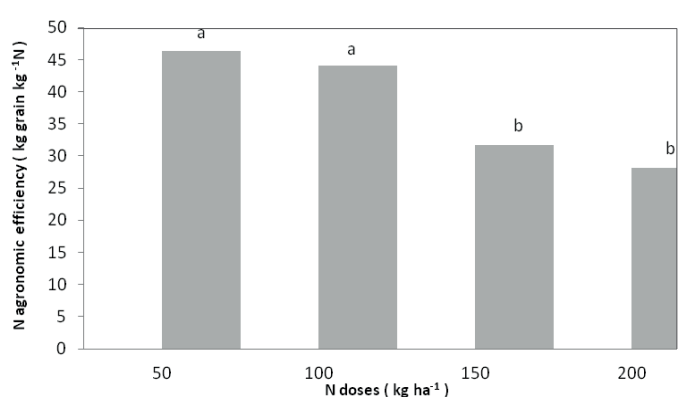

Figure 3. Nitrogen agronomic efficiency (NAE) in twenty rice genotypes with five doses of $\mathrm{N}$ in an Acuic Durixererts soil in Parral, Chile. Different letters indicate significant differences $(\mathrm{P} \leq 0.05)$ according to a LSD test. LSD: least significant difference.

had high NAE and yield components that may be of use when developing new cultivars. Our results demonstrate the importance of selecting rice genotypes with greater NAE and the consideration of this parameter when making $\mathrm{N}$ fertilizer recommendations.

\section{Acknowledgements}

This work was supported by the Rice Breeding Program at the Instituto de Investigaciones Agropecuarias, Centro Regional de Investigación Quilamapu, and the Facultad de

Agronomía, Universidad de Concepción (Chillán, Chile).

\section{Resumen}

C. Quezada, S. Hernaíz, I. Vidal, R. Alvarado, R. Gallegos y W. Yañez. 2013. Selección de genotipos de arroz (Oryza sativa $\mathrm{L}$.) con alta eficiencia agronómica de nitrógeno en un suelo Acuic Durixererts, en la zona centro - sur de Chile. Cien. Inv. Agr. 40(2): 375-385. La eficiencia agronómica de nitrógeno (EAN) es un factor importante que no ha sido considerado en los programas de mejoramiento del cultivo de arroz. El objetivo de esta investigación fue evaluar la eficiencia agronómica del nitrógeno en veinte genotipos de arroz en un suelo Acuic Durixererts (Serie Quella), ubicado en la zona centro sur de Chile ( $\left.36^{\circ} 04^{\prime} \mathrm{S}, 72^{\circ} 0^{\prime} \mathrm{W}\right)$ durante la temporada 2008-2009. El diseño experimental fue de bloques completos al azar con arreglo de parcelas divididas, conformado por cinco tratamientos de dosis de nitrógeno de 0, 50, 100, 150 y $200 \mathrm{~kg} \mathrm{ha}^{-1}$ y veinte genotipos de arroz. Se evaluó rendimiento y sus componentes, características agronómicas, porcentaje de grano lleno y EAN. El nivel de nitrógeno tuvo 
un efecto significativo en rendimiento de grano, EAN y número de panículas por $\mathrm{m}^{2}$. Los genotipos presentaron diferencias altamente significativas en todos los parámetros evaluados. La interacción Nitrógeno $\times$ Genotipo tuvo efecto significativo en altura de plantas, peso de 1.000 granos y porcentaje de grano lleno. Los genotipos de arroz con mejor comportamiento fueron Quila 233006, Quila 223202, CINIA 857 y RQuila17, con dosis de $50 \mathrm{~kg} \mathrm{~N} \mathrm{ha}^{-1}$ y Ámbar, RQuila 17 y Quila 242105 , con dosis de $100 \mathrm{~kg} \mathrm{~N}^{-1}$, por presentar alta EAN y componentes de rendimiento destacados para producir nuevas variedades. Los resultados demuestran la importancia de seleccionar genotipos de arroz con mayor EAN y considerar este parámetro en las recomendaciones de fertilización nitrogenada.

Palabras clave: Dosis nitrógeno, eficiencia agronómica del nitrógeno, genotipos de arroz.

\section{References}

Ahmad, A., I. Khan, Y.P. Abrol, and M. Iqbal. 2008. Genotypic variation of nitrogen use efficiency in Indian mustard. Environmental Pollution 154:462-472.

Alvarado, J.R., and S. Hernaíz. 2007a. Variedades, siembra, semilla certificada, dosis de semillas y épocas de siembra. In: J.R. Alvarado (ed.). Arroz, Manejo Tecnológico. Instituto de Investigaciones Agropecuarias. Chillán, Chile. p. 21-37.

Alvarado, J.R., and S. Hernaíz. 2007b. Calidad industrial del grano. In: J.R. Alvarado (ed.). Arroz, Manejo Tecnológico. Instituto de Investigaciones Agropecuarias. Chillán, Chile. p. 39-48.

Belder, P., B.A.M. Bouman, R. Cabangon, L. Guoan, E.J.P. Quilang, L. Yuanhua, J.H.J. Spiertz, and T.P Tuong. 2004. Effect of water-saving irrigation on rice yield and water use in typical low land conditions in Asia. Agricultural Water Management 65:193-210.

Bond, J., T. Walker, B. Ottis, and D. Harrell. 2008. Rice seeding and nitrogen rate effects, yield and yield components of two rice cultivars. Agronomy Journal 100:393-397.

Cassman, K.G. S. Peng, D.C. Olk, J.K. Ladha, W. Reichart, A. Dobermann, and U. Singh. 1998. Opportunities for increased nitrogen-use efficiency from improved resource management in irrigated rice systems. Field Crops Research 56:7-39.

Chuan, L., P.He, M.P. Pampolino, A.M. Johnston, J. Jin. X. Xu, S. Zhao, S. Qiu, and W. Zhou. 2013. Establishing a scientific basis for fertilizer recommendations for wheat in China: Yield re- sponse and agronomic efficiency. Field Crops Research 140:1-8.

Duan, Y.H., Y.L. Zhang, L.T.Ye, G.H. Xu, and Q.R. Shen. 2007. Responses of rice cultivars with different nitrogen use efficiency to partial nitrate nutrition. Annals of Botany 99:1153-1160.

Fageria, N., and M.P. Barbosa. 2001. Nitrogen use efficiency in lowland rice genotypes. Communications in Soil Science and Plant Analysis 32:2079-2089.

Fillery, I.R., P.A. Roger, and S.K. De Data. 1986. Ammonia volatilization from nitrogen sources applied torice fields. II. Floodwater properties and submerged photosynthetic biomass. Soil Science Society of America Journal 50:86-91.

Haefele, S.M., S.M.A. Jabbar, J.D.L.C. Siopongco, A. Tirol-Padre, S.T. Amarante, P.C. Sta Cruz, and W.C. Cosico. 2008. Nitrogen use efficiency in selected rice (Oryza saliva L.) genotypes under different water regimes and nitrogen levels. Field Crops Research 107:137-146.

Hirzel, J. 2007. Nutrición y fertilización del cultivo de arroz. In: J.R. Alvarado (ed.). Arroz, Manejo Tecnológico. Instituto de Investigaciones Agropecuarias. Chillán, Chile. Boletín No 162. p. 87-64.

Hossain, M.F., S.K. White, S.F. Elahi, N. Sultana, M.H.K. Choudhury, O.K. Alam, J.A. Rother, and J.L. Gaunt 2005. The efficiency of nitrogen fertilizer for rice in Bangladesh farmers' fields. Field Crops Research 93:94-107.

Inthapanya, P., Sipaseuth, P. Sihavong, V. Sihathep, M. Chanphengsay, S. Fukai, and J. Basnayake. 2000. Genotype differences in nutrient uptake and utilisation for grain yield production of rainfed lowland rice under fertilised and non fertilised condictions. Field Crops Research 65:57-68. 
Jiang, L., T. Dai., D. Jiang, W. Cao, X. Gan., and S. Wei, S. 2004. Characterizing physiological Nuse efficiency as influenced by nitrogen management in three rice cultivars. Field Crops Research 88:239-250.

Jian-feng, Ch., J. Han-yan, L. Yi-bai, D. Ting-bo, and C. Wei-xing. 2011. Methods on identification and screening of rice genotypes with high nitrogen efficiency. Rice Science 18:127-135.

Kato, Y., M. Okami, and K. Katsura. 2009. Yield potential and water use efficiency of aerobic rice (Oryza sativa L.) in Japan. Field Crops Research 113:328-334.

Khurana, H.S., A. Bijay-Singh, S.B. Dobermann, S.B. Phillips, A.S. Sidhu, and Y. Singh. 2008. Site-specific nutrient management performance in a rice-wheat cropping system. Better Crops 92:26-28.

Koutroubas, S.D., and D.A. Ntanos. 2003. Genotypic differences for grain yield and nitrogen utilization in Indica and Japonica rice under Mediterranean conditions. Field Crops Research 83:251260.

Ladha, J.K, Kirk, G.J.D., Bennet, J., Peng, S., Reddy, C.K., Reddy, P.M., and Singh, U. 1998. Opportunities for increased nitrogen-use efficiency from improved lowland rice germoplasm. Field Crops Research 56:41-71

Mahajan, G., B.S. Chauhan, J. Timsina, P.P. Singh, and K. Singh. 2012. Crop performance and water-and nitrogen-use efficiencies in dry-seded rice in response to irrigation and fertilizer amounts in northwest India. Field Crops Research 134:59-70.

Ohnishi, M., T. Horie, K. Homma, N. Supapoj, H. Takano, and S. Yamamoto. 1999. Nitrogen management and cultivar effects on rice yield and nitrogen use efficiency in Northeast Thailand. Field Crops Research 64:109-120.

Peng, S., R.J. Buresh, J. Huang, J. Yang, Y. Zou, X. Zhong, G. Wang, and F. Zhang. 2006. Strategies for overcoming low agronomic nitrogen use efficiency in irrigated rice systems in China. Field Crops Research 2006:37-47.

Peng, X., Y. Liu, S. Luo, L. Fan, T. Song, and Y. Guo. 2007. Effects of site-specific nitrogen manage- ment on yield and dry matter accumulation of rice from cold areas of Northeastern China. Agricultural Sciences in China 6:715- 723.

Poshtmasari, H.K., H. Pirdashti, M. Nasiri, and M.A. Bahmanyar. 2007. Chlorophyll content and biologiocal yield of modern and old rice cultivars in different urea fertilizer rates and applications. Asian Journal of Plant Sciences 6:177-180.

Pozo, A. del y P. del Canto. 1999. Áreas agroclimáticas y sistemas productivos en la VII y VIII regiones. Serie Quilamapu No113. INIA Quilamapu. Chillán, Chile.

Raun, W.R., and G.V. Johnson. 1999. Improving nitrogen use efficiency for cereal production. Agronomy Journal 91:357-363.

Sun, Y., J. Ma, Y. Sun, H. Xu, Z. Yang, S. Liu, X. Jia, and H. Zheng. 2012. The effects of different water and nitrogen managements on yield and nitrogen use efficiency in hybri rice of China. Field Crops Research 127:85-98.

Samonte, S.O.P.B., L.T. Wilson, J.C.Medley, S.R.M. Pinson, A.M. McClung, and J.S. Lales. 2006. Nitrogen utilization efficiency. Relationships with grain yield, grain proteins, and yield-related traits in rice. Agronomy Journal 98:168-176.

Singh, U., J. Ladha, E. Castillo, G. Punzalan, A. Tirol-Padre, and M. Duquesa. 1998. Genotypic variation in nitrogen use efficiency in mediumand long-duration rice. Field Crops Research 58:35-53.

Stolpe, N. 2006. Descripciones de los principales suelos de la VIII Región de Chile. Universidad de Concepción. Facultad de Agronomía. Chillán, Chile.84 pp.

Timsina, J., U. Singh, M. Badaruddi, C. Meisner, and M.R. Amin 2001. Cultivar, nitrogen, and water effects on productivity, and nitrogen-use efficiency and balance for rice-wheat sequences of Bangladesh. Field Crops Research 72:143-161.

Tirol-Padre, A., J.K. Ladha, U. Singh, E. Laureles, G. Puinzalan, and S. Akita. 1996. Grain yield performance of rice genotypes at suboptimal levels of soil $\mathrm{N}$ as affected by $\mathrm{N}$ uptake and utilization efficiency. Field Crops Research 46:127-143.

Wei, D., K. Cui, J. Pan, G. Ye, J. Xiang, L. Nie, and J. Huang. 2011. Genetic dissection of grain nitro- 
gen use efficiency and grain yield and their relationship in rice. Field Crops Research 124:340346.
Zhao, S., X. Zhao, and W. Shi. 2012. Genotype variation in grain yield response to basal $\mathrm{N}$ fertilizer supply among different rice cultivars. African Journal of Biotechnology 11:12298-12304. 